\title{
Cilioretinal Artery Occlusion after Endoscopic Sinus Surgery: A Rare and Hardly Remembered Complication
}

\author{
Renata Bigolin Siviero, $M D^{1}$, Carlos A. Moreira-Neto, PhD ${ }^{1}$, Marcello de Quadros Ribeiro, MD', Luiz \\ Gustavo Nocera, $M D^{1}$, Rafaela Bigolin Siviero, $M D^{1}$, Thiago Sasso Carmona de Souza, $M D^{2 *}$, Guilherme \\ Simas do Amaral Catani, PhD², Rogério Hamerschmidt, PhD', Carlos A. Moreira Jr., PhD ${ }^{1}$ \\ ${ }^{1}$ Hospital de Olhos do Paraná, Curitiba, Brazil \\ ${ }^{2}$ Department of Otolaryngology and Head and Neck Surgery - Hospital de Clínicas Complex, Federal \\ University of Paraná, Curitiba, Brazil

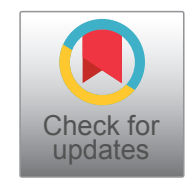

\begin{abstract}
The proximity between nasal and sinus structures and the orbit causes ocular complications to occur with some frequency in endoscopic sinus surgery (ESS). Among them, vascular ones are rare. Only 3 cases of post-ESS retinal artery obstruction have been published to date. Despite the low frequency, arterial occlusions can cause irreversible damage to vision. In the present report, we present a case of a patient who evolved with occlusion of the cilioretinal artery, leading to scotoma formation in the left eye, after ESS. The present report highlights the importance of the otolaryngologist's knowledge of this complication, which, although rare, has high morbidity.
\end{abstract}

Keywords

Intranasal Surgery, Cilioretinal artery occlusion, Cilioretinal artery

\section{Introduction}

For more than 25 years, endoscopic sinus surgery (ESS) has been a relatively safe and efficient method for treating nasal and sinus diseases [1,2]. However, the anatomic closeness and connections among the paranasal sinus structures, nasal cavity, and orbit, make the orbital region vulnerable to intraoperative trauma and to postoperative complications [2].

Ophthalmic complications include direct trauma to the lamina papyracea, tear drainage duct lesion, extrinsic ocular muscle retro-orbital hemorrhages, direct lesions/trauma to the optic nerve, or visual impairment $[1,2]$.

Retinal artery occlusions in ESS, although rare, are severe. The literature includes three cases of central retinal artery occlusion resulting from ESS. Two cases had retro-orbital hemorrhages associated with central retinal artery occlusion, while the third one had an occlusion of the retinal artery due to a fat embolism [1,3].

The purpose of this report is to describe an extremely rare case of cilioretinal artery occlusion after ESS.

\section{Case Presentation}

A 35-year-old male sought a tertiary health center emergency due to a paracentral scotoma in the left eye that began immediately after an ESS that was performed 4 days before in another health center. The ESS description mentioned local infiltration with adrenaline and xylocaine $(1: 80,000)$ into the nasal septum and inferior turbinates during the surgical procedure. The patient did not report comorbidities or previous history of ophthalmological disease.

The visual acuity was 20/20 in the righteye and 20/25 in the left eye. However, the patient reported a paracentral scotoma in the left eye. The dilated fundus appeared normal in the right eye; in the left eye, the papillomacular bundle was very pale and the fovea was spared (Figure $1 \mathrm{~A}$ ).

Since the patient was allergic to iodine, optical coherence tomography angiography confirmed the cilioretinal artery oc-

\footnotetext{
*Corresponding author: Thiago Sasso Carmona de Souza, Department of Otolaryngology and Head and Neck Surgery - Hospital de Clínicas Complex, Federal University of Paraná, R. Gen. Carneiro, 181 - Alto da Glória, Curitiba, Paraná, 80060-900, Brazil, Tel: +55-41-999997892

Accepted: February 06, 2021

Published online: February 08, 2021

Citation: Siviero RB, Neto CAM, Ribeiro MQ, et al. (2021) Cilioretinal Artery Occlusion after Endoscopic Sinus Surgery: A Rare and Hardly Remembered Complication. Otolaryngol Res Rev 4(1):66-68
} 


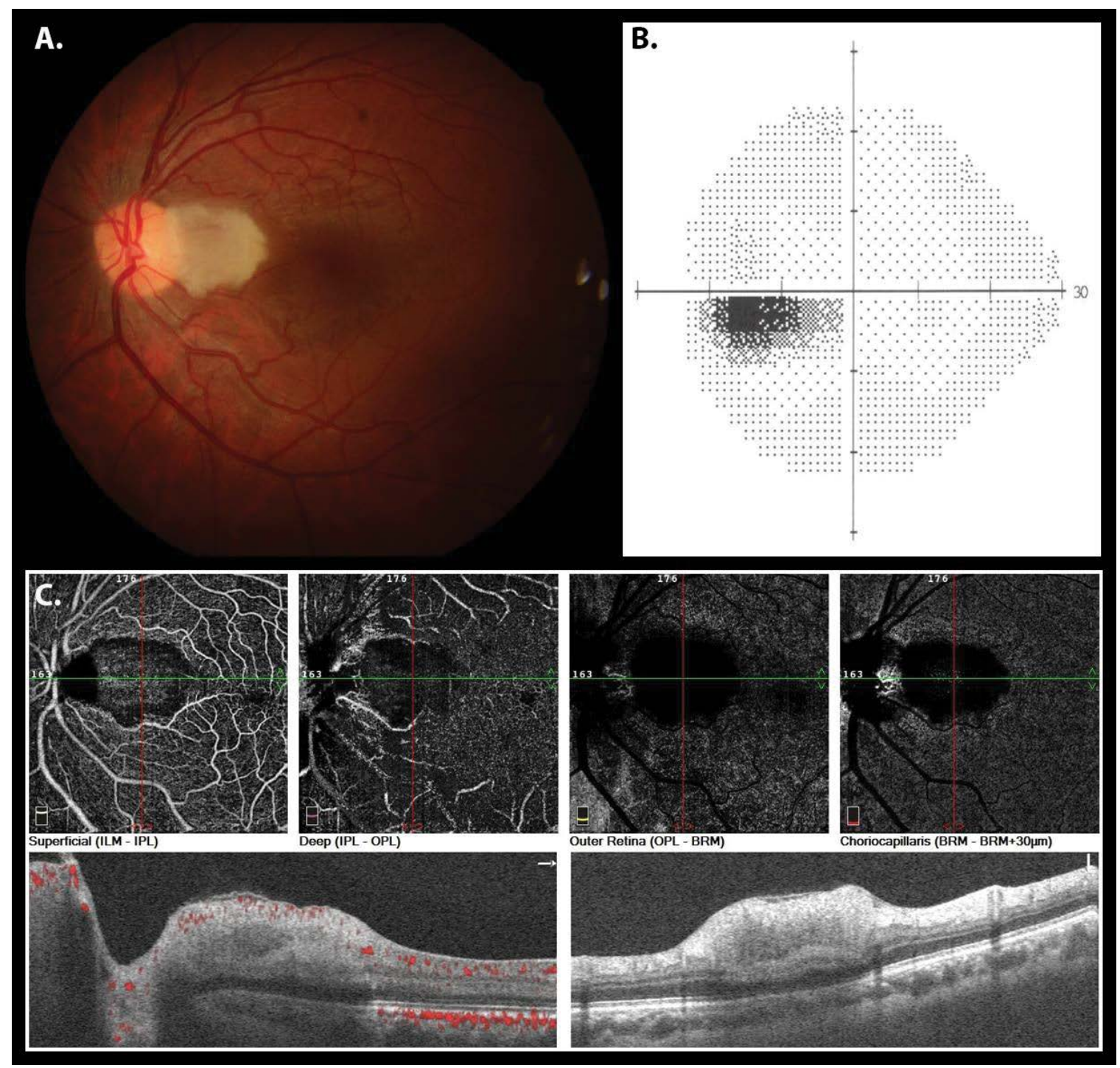

Figure 1: (A) Left eye of a 35-year-old man. A color fundus photograph shows a pale area in the papillomacular bundle; (B) Computerized perimetry shows a centrocecal scotoma in the left eye; (C) Optical coherence tomography angiography does not show blood flow in either the superficial or deep plexus in the pale area.

clusion in the left eye (Figure 1C). Computerized perimetry (Humphrey Visual Perimetry, Estimulus III; Carl Zeiss, Germany, 24-2) was normal in the right eye and showed a centrocecal defect in the left eye (Figure 1B).

The medical team chose not to carry out any intervention, due to the late presentation of the patient, with more than 100 hours after symptom onset.

\section{Discussion}

Retinal artery occlusions are rare complications in ESS. To date, only three reports of such complications have been published, and all were described as central retinal artery or branch retinal artery occlusions [1-3]. An extensive literature search was performed by the authors and no case of a cilioretinal artery occlusion after ESS was found. Thus, to the best of our knowledge, the current case is the first to report this ESS complication.

The cilioretinal arteries are part of the posterior ciliary artery system and are present in approximately $20 \%$ to $30 \%$ of the population [4]. Cases of retinal artery occlusion that spares the cilioretinal artery are well documented in the literature [5].

ESS is a term used to refer to a group of surgical procedures performed through endoscopic vision to treat nasal and sinuses diseases $[1,2]$. The procedure provides better visibility of structures, requires less surgical manipulation, and is associated with lower complication rates. For these reasons, ESS has gained popularity among otolaryngologists and its indications include a wide list of pathologies (e.g. chronic rhinosinusitis, mucoceles, orbital decompression) [1-4]. The close relation between sinonasal structures and the orbital cavity makes the orbit and its contents potentially susceptible to complications [1-3].

Ophthalmologic complications during ESS, as direct trauma to the lamina papyracea, direct lesion to the optic nerve, retro-orbital hematoma and arterial occlusion, can cause ir- 
reversible visual damage and affect patients' quality of life. Artery occlusions (retinal or cilioretinal) caused by nasal surgeries, although extremely rare, is one of the worst complications, because there are few effective treatments and the visual prognosis is usually poor $[2,3]$.

In most cases, the presence of the cilioretinal artery functions as a protective factor against occlusions [4]. However, in the reported case, the patient had left eye cilioretinal artery exclusive occlusion after an ESS, resulting in a paracentral scotoma. Once genetic causes and thrombophilia were excluded, two possible etiologies for the occlusion were considered: Fat embolism resulting from excessive surgical handling (this was considered less probable), and vasospasms resulting from local (nasal septum and turbinates) adrenaline infiltration. The adrenaline concentration infiltrated during surgery $(1: 80,000)$ is considered safe. However, in rare cases, the adrenaline can penetrate inadvertently into the blood vessels, causing vasospasms and arterial occlusion.

Regardless the cause, delays in identifying the occlusion, either due to diagnostic difficulties or due to the patient's delay in seeking medical evaluation, significantly compromise the prognosis. The rapid death of the ischemic retinal cells often limits the treatment. Therefore, it is extremely important that the otolaryngologist is aware of this rare, but possible ESS complication. A cautious and controlled intraoperative adrenaline infiltration combined with orientations to the pa- tient regarding postoperative ophthalmic complaints can prevent unfavorable outcomes. Likewise, the ophthalmologist's knowledge of this possible ESS complication can speed up the diagnosis, enabling early treatment.

\section{Acknowledgements}

We would like to thank our department colleagues.

\section{Conflicts of Interest}

None declared.

\section{References}

1. Siedek V, Pilzweger E, Betz C, et al. (2013) Complications in endonasal sinus surgery: a 5 -year retrospective study of 2,596 patients. Eur Arch Otorhinolaryngol 270: 141-148.

2. Seredyka-Burduk M, Burduk PK, Wierzchowska M, et al. (2017) Ophthalmic complications of endoscopic sinus surgery. Braz $\mathrm{J}$ Otorhinolaryngol 83: 318-323.

3. Kubo N, Ono A, Nishimura T, et al. (2003) Onset of central retinal artery occlusion during the endoscopic sinus surgery. Pract Oto-Rhino-Laryngol 96: 697-703.

4. Jackson E (1911) Cilio-retinal and other anomalous retinal vessels. Ophthalmic Rev 30: 264-269.

5. Hayreh SS, Zimmerman MB (2005) Central retinal artery occlusion: visual outcome. Am J Ophthalmol 140: 376-391.

DOI: $10.36959 / 926 / 555$

Copyright: (C) 2021 Siviero RB, et al. This is an open-access article distributed under the terms of the Creative Commons Attribution License, which permits unrestricted use, distribution, and reproduction in any medium, provided the original author and source are credited. 